\title{
Undifferentiated Small Round Cell Sarcoma of the Postcricoid Region of the Hypopharynx: A Rare Case Report and Review of the Literature
}

\author{
Jiao Zhu' \\ Bo $\mathrm{Li}^{\mathrm{I}}$ \\ Juan Juan $\mathrm{Hu}^{\prime}$ \\ Weiping Liu ${ }^{2}$ \\ Dan Lu' \\ Jun Liu'
}

'Department of Otorhinolaryngology Head \& Neck Surgery, West China Hospital, Sichuan University, Chengdu, People's Republic of China; ${ }^{2}$ Department of Pathology, West China Hospital, Sichuan University, Chengdu, People's Republic of China
Correspondence: Dan Lu; Jun Liu Department of Otorhinolaryngology Head \& Neck Surgery, West China Hospital, Sichuan University, Wuhou Distrcit, Chengdu, People's Republic of China

Email dashijie00I@I63.com;

hxheadneckjunl@I63.com

\begin{abstract}
Undifferentiated small round cell sarcoma (USRCS) is a rare malignant tumor. No reports about USRCS of the postcricoid region of the hypopharynx are available, and its clinical features and treatment guidelines remain undefined. We report a case of USRCS of the postcricoid region of the hypopharynx with treatment of surgery and radiotherapy. A 50year-old man presented with a $4.9 \mathrm{~cm} \times 3.7 \mathrm{~cm}$ tumor in the postcricoid region of the hypopharynx. It was diagnosed as USRCS. The tumor showed small round cells and positive immunoexpression of CD56, Cyclin D1, TLE1 and CD99, but no rearrangement or fusion of EWSR1, SS18, CIC, and BCOR. The patient underwent radiotherapy after surgery, without signs of tumor progression and metastasis at the 9-month follow-up. In conclusion, USRCS of postcricoid region of hypopharyngeal is ultra-rare. To make diagnosis, pathological examination is necessary. Surgery followed by radiotherapy might offer an optional treatment for USCRS.
\end{abstract}

Keywords: hypopharynx, sarcoma, immunohistochemistry, case report

\section{Introduction}

Undifferentiated small round cell sarcoma (USRCS) is a rare, highly malignant tumor that mainly affects soft tissues and bones. It has a poor prognosis. In the World Health Organization (WHO) classification of soft tissue tumors and bone tumors published in 2020, USRCS has been introduced as a new chapter, ${ }^{1}$ which includes Ewing sarcoma and three other sarcoma subsets: 1) round cell sarcomas with EWSR1 fusion to non-ETS family members, 2) CIC-rearranged sarcomas, and 3) BCOR-rearranged sarcomas. ${ }^{2}$ Though most cases of USRCS can be accurately classified using a combination of morphological, immunohistochemical, and molecular findings, a few rare cases cannot be classified into the existing categories and require further research.

Malignancies of the hypopharynx are relatively rare and account for less than 3\% of all head and neck tumors. The majority of hypopharyngeal malignancies are squamous cell carcinomas, ${ }^{3}$ and the other diagnoses include adenocarcinoma, undifferentiated carcinoma, neuroendocrine neoplasm, sarcoma and so on. Anatomically, the hypopharynx includes the lateral pharynx, posterior pharyngeal wall, piriform sinuses, and the postcricoid region. To our knowledge, no reports about USRCS of the postcricoid region of the hypopharynx are available. Herein, we report a unique case of a 50-year-old man with USRCS along with a literature review of some similar previous cases. 


\section{Case Report}

\section{Clinical Characteristics}

A 50-year-old man presented with a 6-month history of progressive symptoms of foreign body sensation and slight hoarseness without tightness in breathing. He had not visited a hospital until he experienced worsening dysphagia, which lasted for 1 month. He denied any history of a pharyngeal foreign body, hyperventilation, hemoptysis, or laryngopharyngeal reflux. His previous medical history included surgical treatment for esophageal fibroma 3 years ago.

Fiberoptic laryngoscopy showed a large smooth nodular mass in the postcricoid region of the hypopharynx (Figure 1A). No abnormal blood vessels were observed on narrowband imaging (NBI). High-resolution computed tomography (HRCT) of the neck demonstrated a large soft tissue lesion located in the hypopharynx with a maximum cross-sectional area of $4.9 \mathrm{~cm} \times 3.7 \mathrm{~cm} \times 3.1 \mathrm{~cm}$, without any bone erosion. The mass showed heterogeneous enhancement on contrast HRCT (Figure 1B and C). The HRCT also showed increased submandibular lymph nodes and bilateral lymphadenopathy of the carotid sheath. Results of the auxiliary examinations, including electrocardiogram, and the laboratory data were normal. In general, there was no conclusive evidence of distant metastases according to chest HRCT, abdomen sonography, and gastroscopy.

\section{Treatment and Outcome}

Because the patient had experienced esophageal fibroma and the surface of the tumor was smooth, fibroma was considered as the preliminary diagnosis. A surgery was performed using the suprahyoid approach. Intraoperatively, a round, pale yellow, and smooth mass with a thin capsule was clearly observed, and it pushed the posterior annulus into the esophagus. During surgery, frozen-tissue analysis was performed for biopsy, and its findings indicated mitosis in the tumor. Therefore, the pathological diagnosis was a round or ovoid tumor. However, it was difficult to characterize the tumor as benign or malignant. Therefore, the tumor was removed completely without an expanded margin (Figure 2A-C).

One week after the surgery, the patient was discharged without any complications. After the surgery, he was fed using a nasogastric tube until the restoration of the surgical field 3 weeks later, when he underwent a reexamination via fiberoptic laryngoscopy (Figure $3 \mathrm{~A}$ and B). Then, he could eat normally without dysphagia, hoarseness, or choking cough. The patient received postoperative radiotherapy (a total of 60 Gy in two fractions) 4 weeks after the surgery. There were no definite signs of tumor progression at the latest follow-up more than 9 months after the diagnosis of USRCS (Figure 3C).
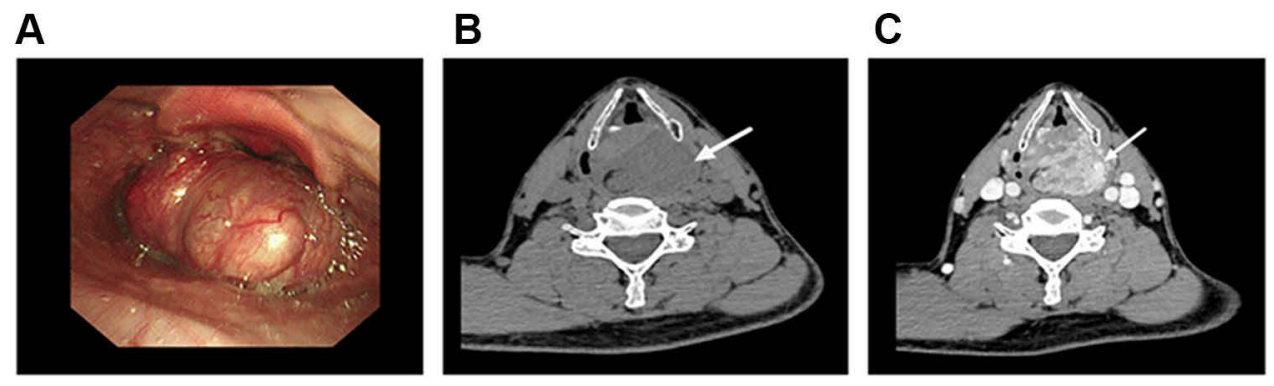

Figure I Preoperative images of this patient. (A) laryngoscopic image. (B) CT without contrast. (C) CT with contrast.

A

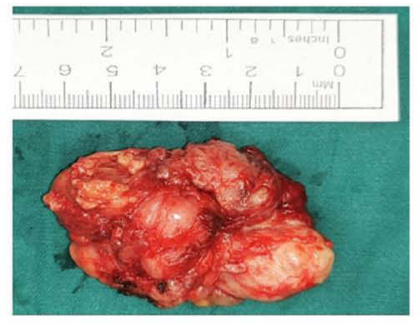

B

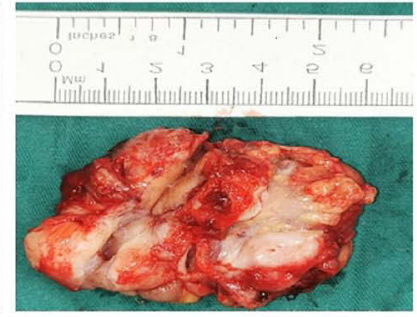

C

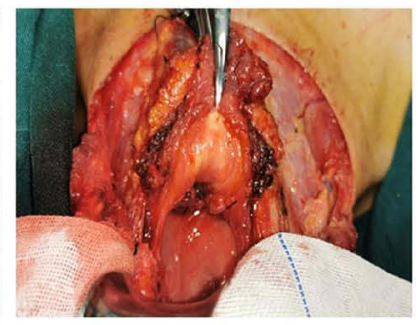

Figure 2 Intraoperative images of this patient. (A) the tumor presented as an oval, pale yellow, smooth mass with a thin envelope. (B) the mass was a solid tumor. (C) Postoperative field with smooth mucosa. 
A

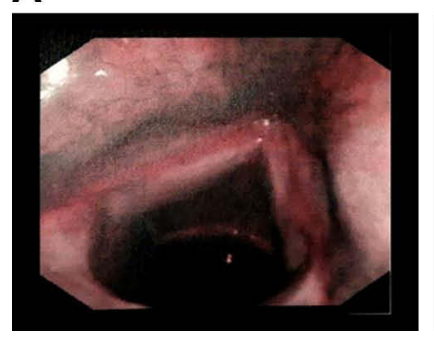

B

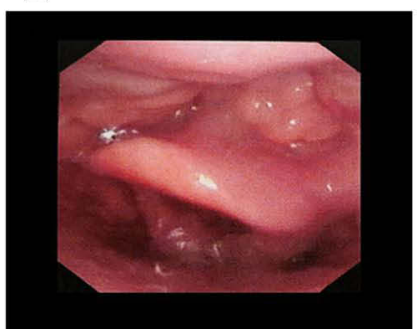

C

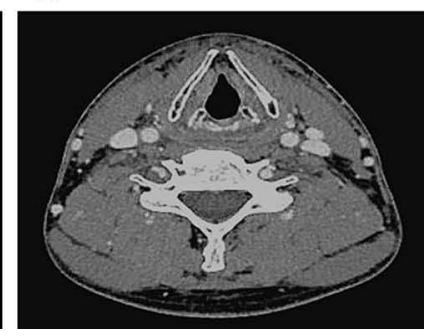

Figure 3 Follow-up images of this patient. (A) follow-up laryngoscopic image under NBI. (B) follow-up laryngoscopic image. (C) follow-up CT scans with contrast.

\section{Histopathological Findings}

Hematoxylin and eosin-stained sections of excised lesion tissue from the patient showed a nodular neoplasm, comprising sheet-like and ill-defined small round blue cells with hypochromatic cytoplasm and hyperchromatic nucleus. The tumor stroma was fibrous and collagenous. Microvascular proliferation and a few pyknotic nuclei were observed, but other types of cells, such as spindle cells, and nuclear division were not observed (Figure 4A).

Furthermore, Immunohistochemistry (IHC) was performed by staining the formalin-fixed paraffin-embedded (FFPE) tissues with corresponding primary antibodies (Supplementary Table 1), which were incubated at $4{ }^{\circ} \mathrm{C}$ overnight. Strong immune expression of CD56, Cyclin D1 and TLE-1 (Figure 4B-D) with a high proliferation index (Ki-67: $50 \%$ ) (Figure $4 \mathrm{E}$ ) were observed by using the microscope (Zeiss, Germany). Moreover, focal positivity for p53 (Figure 4F), partial positivity for CD99 (Figure 4G) and complete negativity for PCK, CK7, p63, CK5/6, S-100, Des, SMA, CD34, STAT-6, myogenin, and myoD1 were observed.

Fluorescence in situ hybridization (FISH) analysis was performed on 4-um-thick formalin-fixed paraffin-embedded tissue sections. Corresponding break- apart probes were used for the EWSR1 (Vysis EWSR1 Break Apart FISH Probe Kit;
Abbott Molecular, Abbott Park, USA), SS18 (Vysis SS18 Break Apart FISH Probe Kit; Abbott Molecular, Abbott Park, USA), CIC (custom-made probe; Chromosome Science Labo, Japan) and BCOR (custom-made probe; Chromosome Science Labo, Japan). The FISH images, captured by using of Clinical Cytogenetics Software \& Instruments: Leica Biosystems, were assessed by two independent operators. The positive threshold for calling the FISH assay positive was $15 \%$. We did not observe any rearrangement or fusion of EWSR1, SS18, CIC, and BCOR. In all, these findings from morphological, immunohistochemical, and molecular genetic analyses support the diagnosis of USRCS.

\section{Discussion}

USRCS is a relatively rare tumor with an incidence rate of approximately 0.1 cases per 100,000 population, ${ }^{2}$ and no reports about USRCS of the hypopharynx are available. According to the WHO classification of tumors, undifferentiated sarcomas can be broadly divided into pleomorphic, spindle cell, round cell, and epithelioid subsets. ${ }^{4}$ Classified as undifferentiated sarcoma, USRCS is a heterogeneous group of tumors mostly originating from the mesoderm and having a poor prognosis. ${ }^{5}$ Many USRCSs can now be more precisely classified into specific

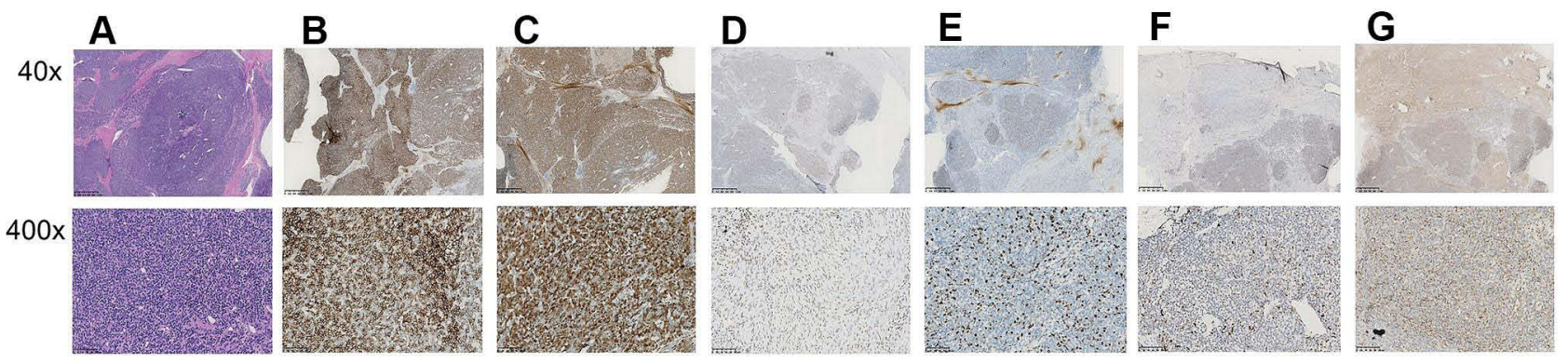

Figure 4 Histopathological findings. (A) The view of the tumor tissue slice (Hematoxylin-eosin stain). Nodular neoplasm with hyperchromatic nucleus, small blue round cells with hypochromatic cytoplasm, collagenization of tumor stroma, microvascular proliferation and a few pyknotic nuclei were observed (Original magnification $\times 40$, x400). (B-G) Immunohistochemical staining of positive expression (Original magnification x40, x400). (B) Intense positive expression of CD56, (C) strong positive expression of Cyclin DI, (D) strong positive expression of TLE-I, (E) the over expressed protein of Ki-67 (50\%), (F) focal positive expression of P53, (G) partial positive expression of CD99. 
molecular subsets. However, owing to the lack of an identifiable line of differentiation, approximately $10-20 \%$ of soft tissue sarcomas still cannot be classified. ${ }^{6}$ The USRCS case reported herein is an extremely rare case.

The clinical diagnosis of USRCS of the hypopharynx is extremely difficult because of the nonspecific clinical course and the atypical findings on fiberoptic laryngoscopy and imageological examination. Patients with hypopharyngeal tumors may be asymptomatic until laryngeal invasion, following which they may present with sore throat and/or hoarseness or nodal metastasis with a neck lump. ${ }^{3}$ Symptoms in early stages of hypopharyngeal tumors are nonspecific and may imitate benign conditions such as foreign body sensation or laryngopharyngeal reflux. ${ }^{3}$ In this case, clinical course of the disease initiated with the symptom of foreign body sensation and gradually developed into dysarthria and dysphagia, consistent with the clinical presentation of hypopharyngeal tumors.

Besides the medical history and physical examination, comprehensive pharynx, larynx, and neck examinations, including fiberoptic laryngoscopy and imaging, are necessary to make a prompt diagnosis for hypopharyngeal tumors. ${ }^{3}$ The shape, color, location, size, and boundary of the tumor can be observed directly using fiberoptic laryngoscopy to some extent. Moreover, NBI, as an innovative endoscopic technology, can detect the potential focal increase in vascularization and abnormal angiogenetic changes of the superficial mucosa. ${ }^{7}$ In recent years, NBI has been widely used to detect lesions of the pharynx and larynx. Currently, the main benefits provided by NBI are 1) the early diagnosis of tumors, 2) the screening of cancers, 3 ) the surveillance of patients who are already undergoing treatments, and 4) the optimal definition of the intraoperative surgical margins. ${ }^{8}$ In addition, Di Maio et $\mathrm{al}^{9}$ reported that the sensitivity and specificity of NBI for the detection of occult mucosal tumors in patients with head and neck squamous cell carcinoma of unknown primary origin were 0.83 and 0.88 , respectively. While the sensitivity and specificity of NBI in the diagnosis of hypopharyngeal sarcoma have not been reported, the application of NBI for screening or diagnosis of the tumor was not useful in the present case. In our patient, this rare tumor could easily be miscategorized as a benign tumor because of its smooth, round, and well-circumscribed features on fiberoptic laryngoscopy and the negative findings on NBI.

Sonography, as a low-cost and noninvasive assessment of cervical lymph nodes, is one of the imaging modalities recommended for hypopharyngeal cancer, but useless for assessing the primary tumor site. ${ }^{10}$ According to the United Kingdom National Multidisciplinary Guidelines for Head and Neck Cancer 2016, both CT with contrast enhancement and magnetic resonance imaging (MRI) can be used for assessment of the primary site and regional lymphatics. CT can better delineate the bony destruction, even the cortical disruption of ossified cartilage. The advantages of MRI include better soft tissue differentiation and functional imaging ability. ${ }^{11}$ Generally, malignant tumors of uncertain differentiation tend to have aggressive features on MRI. Usually, with careful analysis of radiological information, such as the location, size, tissue layer, and overall anatomical extent including extracompartmental extension and neurovascular invasion, it is possible to make a preliminary judgment about whether the tumor is benign or malignant. In this case, considering the benign characteristics evaluated by fiberoptic laryngoscopy, contrast CT was performed. No special features were observed other than the heterogeneous enhancement without bone erosion, which made it difficult to diagnose the mass. In fact, owing to its excellent tissue contrast and lack of ionizing radiations, MRI is recommended as the ideal modality of choice for the detection, characterization, and local staging of soft tissue tumors. ${ }^{12}$ However, because of its nonspecial and overlapping features, it is still difficult to diagnose USRCS. Yoo et $\mathrm{al}^{13}$ reported that a notable feature of undifferentiated sarcomas is "a tail sign," which is present as thick fascial enhancement extending from the sarcoma margin. Moreover, "a tail sign" is associated with locoregional recurrence to a certain extent. However, owing to limited numbers of cases, there are no large studies investigating the MRIspecific features of different undifferentiated sarcomas, including USRCS. ${ }^{12}$ In addition, positron emission tomography (PET), a valuable tool for characterization of tumor metabolism, is recommended for distinguishing between benign lesions and soft tissue sarcomas based on the uptake of 2-deoxy-2-fluoro-D-glucose. ${ }^{14}$ Moreover, PET is recommended for identification of target regions for biopsy and detection of metastases during the follow-up of treatments for sarcomas. ${ }^{15}$ Although some research has been performed to assess sarcoma using PET, it is still in progress. Thus, PET has not yet been proven as a routine investigative technique for sarcomas. ${ }^{16}$ Because the present case was diagnosed as USRCS, we plan to conduct an MRI examination when necessary during the follow-up. Notably, all the above mentioned factors make the clinical identification of USRCS of the hypopharynx challenging. 
The final diagnosis of USRCS still depends on pathological examination, which relies on a combination of morphology, immunohistochemistry, and molecular techniques. ${ }^{17}$ According to the WHO classification (2020), USRCS comprised Ewing sarcoma and three other main categories of sarcoma, including round cell sarcomas with EWSR1-non-ETS fusions, CIC-rearranged sarcomas, and sarcomas with $B C O R$ genetic alterations. Ewing sarcoma is characterized by canonical fusions between EWSR1 and a gene of the ETS family of transcription factors. Despite considerable morphological overlap among them, these sarcomas tend to present different molecular alterations and clinical outcomes. Moreover, the following round cell sarcomas should be considered in the differential diagnosis of these USRCSs: rhabdomyosarcoma, small cell osteosarcoma, desmoplastic small round cell tumor, mesenchymal chondrosarcoma, and poorly differentiated round cell synovial sarcoma. ${ }^{2}$ Immunophenotypically, sarcoma tissues, deriving from mesenchymal tissue, may express some special markers such as CD56, TLE-1 and Cyclin D1, but not surface the markers of epithelial cells such as CK7, CK5/6 and EMA. In the present case, undoubtedly, the immunohistochemical results were consistent with the above findings. Moreover, the expression of immune markers for cells of neurogenic (S100), muscle cell derived (SMA, DES, Myogenin), interstitial cell derived (STAT-6) and vasogenic (CD 34) were negative, which was great significance for the differential diagnosis. Further, no rearrangements or fusions of genes of EWSR1, CIC, SS18, and BCOR were observed by FISH analyses. So, we made the final diagnosis of USRCS mainly based on the morphology and immunohistochemistry. However, based on the increasingly available molecular techniques, such as PCR and next-generation sequencing, further research for this case will be performed to detect accurate identification of aberrant gene.

Thus far, no specific treatment guidelines for USRCS are available. The common treatment strategies for USRCS mainly include the management of bone sarcoma and soft tissue sarcoma. ${ }^{18}$ According to the guideline, ${ }^{19}$ the cornerstone of treatment is surgical resection of the primary tumor with wide margin, which is typically accompanied by adjuvant radiotherapy and/or chemotherapy. The use of adjuvant radiotherapy or chemotherapy may be determined based on biological risk factors. ${ }^{20}$ More specifically, the aim of surgery is to completely excise the tumor with a commonly accepted margin of $1 \mathrm{~cm}$. Moreover, surgery remains a palliative treatment option for patients with metastatic disease. ${ }^{16}$ Regarding radiotherapy, both pre- and postoperative radiotherapy are considered standard approaches. The recommended doses of pre- and postoperative radiation are 50 Gy and 60-66 Gy, respectively, in 1.8-2 Gy fractions. ${ }^{21}$ Owing to the rarity and heterogeneity of USRCS, it is difficult to demonstrate the efficacy of adjuvant chemotherapy. Therefore, the benefit of adjuvant chemotherapy remains uncertain, despite some studies on this topic. Hence, adjuvant chemotherapy is not routinely recommended. However, when the risk of metastasis is particularly high, the combination of doxorubicin, cisplatin, methotrexate, and ifosfamide is considered. $^{22}$

In the present case, based on the complex situation (mass size, anatomical location, histopathological features, and patient's decision), surgical resection followed by radiotherapy was effective and feasible. When discussing treatment options for hypopharyngeal tumors, laryngeal preservation is an important but controversial issue, and there is still a lack of consensus regarding what and how to achieve functional larynx preservation. Because of the specificity of the anatomical location, expanded excision of the postcricoid region may affect the function of the larynx and swallowing. Therefore, we adopted the suprahyoid approach, which is recommended as a convenient and safe surgical method for full exposure of the postcricoid region of the hypopharynx. Moreover, owing to the intact capsule and basement at the posterior pharyngeal wall, the tumor was excised completely and successfully without expanded margin. Following this organ preservation therapy, the patient had normal and satisfactory respiratory, vocal, and swallowing functions. Because the largest lymph node was less than $1.0 \mathrm{~cm}$ and the tumor could not be distinguished as benign or malignant based on the intraoperative frozen-tissue analysis findings, cervical lymph node dissection was not performed. Postoperative adjuvant radiotherapy and chemotherapy were recommended, but the patient received radiotherapy only. He received a total of 60 Gy of radiation, and is asymptomatic without any signs of recurrence and still being followed up.

In recent years, the use of monoclonal antibodies, cellular therapies, vaccines, and novel treatments such as targeted immunogenic therapies and immunomodulatory therapies has contributed to the development of cancer therapies and improvement of survival rates. 


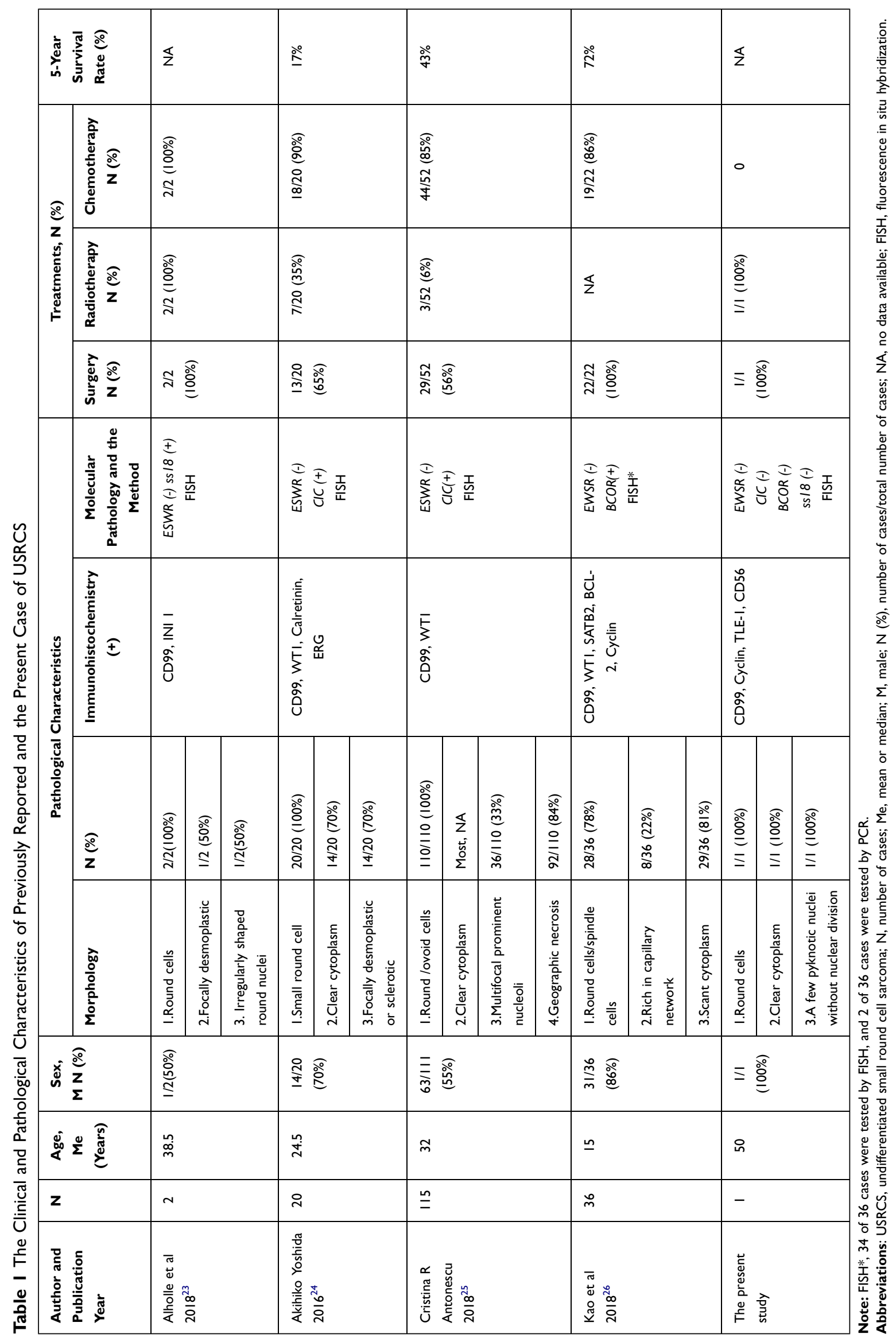


Nevertheless, these treatment methods for USRCS are still at an early stage, and more studies are needed to evaluate their efficacy and side effects. ${ }^{19}$

Moreover, the treatment strategy, effectiveness, and outcome for USRCS are still uncertain. To understand the clinical presentation, morphological spectrum, and outcome, we summarized some previously reported cases diagnosed as USRCS at other sites. ${ }^{23-26}$ The details are presented in Table 1.

In conclusion, this study reports a rare case of USRCS of the postcricoid region of the hypopharynx and describes the clinical and pathological characteristics of some similar cases. Taken together, USRCSs are rare, even ultra-rare, sarcomas. In the present case, the following three features raised the suspicion of USRCS: 1) small round cells with hyperchromatic nucleus and hypochromatic cytoplasm, 2) no distinct line of differentiation, and 3) positive results for specific immunohistochemical markers such as CD99. Moreover, to make a diagnosis, it is necessary to test for the immunohistochemical markers of WT1 and cyclin and molecular genes of EWSR1, SS18, CIC, and BCOR. Despite the absence of specific treatment guidelines, wide surgical excision followed by optimal adjuvant radiotherapy and/or chemotherapy might offer a mode of therapy for reference in cases of USRCS.

\section{Ethics Approval and Consent to Participate}

This research was approved by the Biomedical Ethics Committee of West China Hospital of Sichuan University. The patient provided written informed consent for the publication of her images and case details.

\section{Acknowledgments}

We thank the physicians, nurses, pathologist, oncologist.

\section{Author Contributions}

All authors contributed to data analysis, drafting or revising the article, gave final approval of the version to be published, agreed to the submitted journal, and agree to be accountable for all aspects of the work.

\section{Funding}

This research did not receive any specific grant from funding agencies in the public, commercial, or not-forprofit sectors.

\section{Disclosure}

The authors report no conflicts of interest in this work.

\section{References}

1. De Alava E, Lessnick SL, Stamenkovic I. Ewing sarcoma. In:The who classification of tumors Editorial Board editors. WHO Classification of Tumors Soft Tissue and Bone Tumors. 5th ed. Lyon: IARC Press; 2020:323-325.

2. Sbaraglia M, Bellan E, Dei Tos AP. The 2020 WHO classification of soft tissue tumours: news and perspectives. Pathologica. 2020;11 (3):1-15. doi:10.32074/1591-951X-213

3. Garneau JC, Bakst RL, Miles BA. Hypopharyngeal cancer: a state of the art review. Oral Oncol. 2018;86:244-250. doi:10.1016/j. oraloncology.2018.09.025

4. Dei Tos AP, Mertens F, Pillay N. Undifferentiated sarcoma. In: The WHO Classification of Tumors Editorial Board editors. WHO Classification of Tumours Soft Tissue and Bone Tumours. 5th ed. Lyon: IARC Press; 2020:8-320.

5. Watson S, Perrin V, Guillemot D, et al. Transcriptomic definition of molecular subgroups of small round cell sarcomas. J Pathol. 2018;245(1):29-40. doi:10.1002/path.5053

6. Choi JH, Ro JY. The 2020 WHO classification of tumors of soft tissue: selected changes and new entities. Adv Anat Pathol. 2021;28 (1):44-58. doi:10.1097/PAP.0000000000000284

7. Sun C, Han X, Li X, Zhang Y, Du X. Diagnostic performance of narrow band imaging for laryngeal cancer: a systematic review and meta-analysis. Otolaryngol Head Neck Surg. 2017;156(4):589-597. doi:10.1177/0194599816685701

8. Piazza C, Bon FD, Peretti G, Nicolai P. 'Biologic endoscopy': optimization of upper aerodigestive tract cancer evaluation. Curr Opin Otolaryngol Head Neck Surg. 2011;19(2):67-76. doi:10.1097/ MOO.0b013e328344b3ed

9. Di Maio P, Iocca O, De Virgilio A, et al. Narrow band imaging in head and neck unknown primary carcinoma: a systematic review and meta-analysis. Laryngoscope. 2020;130(7):1692-1700. doi:10.1002/ lary. 28350

10. Fukuhara T, Matsuda E, Hattori Y, et al. Usefulness of ultrasound for assessing the primary tumor of hypopharyngeal carcinoma. Laryngoscope Investig Otolaryngol. 2017;2(6):390-394. doi:10.1002/lio2.126

11. Cho SJ, Lee JH, Suh CH, et al. Comparison of diagnostic performance between CT and MRI for detection of cartilage invasion for primary tumor staging in patients with laryngo-hypopharyngeal cancer: a systematic review and meta-analysis. Eur Radiol. 2020;30 (7):3803-3812. doi:10.1007/s00330-020-06718-8

12. Ahlawat S, Fayad LM. Revisiting the WHO classification system of soft tissue tumours: emphasis on advanced magnetic resonance imaging sequences. Part 1. Pol J Radiol. 2020;85:e396-e408. doi:10.5114/pjr.2020.98685

13. Yoo HJ, Hong SH, Kang Y, et al. MR imaging of myxofibrosarcoma and undifferentiated sarcoma with emphasis on tail sign; diagnostic and prognostic value. Eur Radiol. 2014;24(8):1749-1757. doi:10.1007/s00330-014-3181-2

14. Ioannidis JP, Lau J. 18F-FDG PET for the diagnosis and grading of soft-tissue sarcoma: a meta-analysis. J Nucl Med. 2003;44 (5):717-724. PMID: 12732672.

15. Albano D, Dondi F, Schumacher RF, et al. Clinical and prognostic role of $18 \mathrm{~F}-\mathrm{FDG}$ PET/CT in pediatric Ewing sarcoma. J Pediatr Hematol Oncol. 2020;42(2):e79-e86. doi:10.1097/MPH.00000 00000001518

16. Dangoor A, Seddon B, Gerrand C, Grimer R, Whelan J, Judson I. UK guidelines for the management of soft tissue sarcomas. Clin Sarcoma Res. 2016;6(1):20. doi:10.1186/s13569-016-0060-4 
17. Neuville A, Ranchère-Vince D, Dei Tos AP, et al. Impact of molecular analysis on the final sarcoma diagnosis: a study on 763 cases collected during a European epidemiological study. Am J Surg Pathol. 2013;37(8):1259-1268. doi:10.1097/PAS.0b013e31828f51b9

18. Grünewald TG, Alonso M, Avnet S, et al. Sarcoma treatment in the era of molecular medicine. EMBO Mol Med. 2020;12(11):e11131. doi: $10.15252 / \mathrm{emmm} .201911131$

19. Gómez J, Tsagozis P. Multidisciplinary treatment of soft tissue sarcomas: an update. World J Clin Oncol. 2020;11(4):180-189. doi:10.5306/wjco.v11.i4.180

20. Sundby Hall K, Bruland ØS, Bjerkehagen B, et al. Adjuvant chemotherapy and postoperative radiotherapy in high-risk soft tissue sarcoma patients defined by biological risk factors-A Scandinavian Sarcoma Group study (SSG XX). Eur J Cancer. 2018;99:78-85. doi:10.1016/j.ejca.2018.05.011

21. Gamboa AC, Gronchi A, Cardona K. Soft-tissue sarcoma in adults: an update on the current state of histiotype-specific management in an era of personalized medicine. CA Cancer J Clin. 2020;70 (3):200-229. doi:10.3322/caac.21605
22. Brown HK, Schiavone K, Gouin F, Heymann MF, Heymann D. Biology of Bone Sarcomas and New Therapeutic Developments. Calcif Tissue Int. 2018;102(2):174-195. doi:10.1007/s00223-017-0372-2

23. Alholle A, Karanian M, Brini AT, et al. Genetic analyses of undifferentiated small round cell sarcoma identifies a novel sarcoma subtype with a recurrent CRTC1-SS18 gene fusion. J Pathol. 2018;245 (2):186-196. doi:10.1002/path.5071

24. Yoshida A, Goto K, Kodaira M, et al. CIC-rearranged sarcomas: a Study of 20 cases and comparisons with Ewing sarcomas. Am J Surg Pathol. 2016;40(3):313-323. doi:10.1097/PAS.0000000000000570

25. Antonescu CR, Owosho AA, Zhang L, et al. Sarcomas with CIC-rearrangements are a distinct pathologic entity with aggressive outcome: a clinicopathologic and molecular study of 115 cases. Am J Surg Pathol. 2017;41(7):941-949. doi:10.1097/PAS.0000000000000846

26. Kao YC, Owosho AA, Sung YS, et al. BCOR-CCNB3 fusion positive sarcomas: a clinicopathologic and molecular analysis of 36 cases with comparison to morphologic spectrum and clinical behavior of other round cell sarcomas. Am J Surg Pathol. 2018;42(5):604-615. doi:10.1097/PAS.0000000000000965

\section{Publish your work in this journal}

OncoTargets and Therapy is an international, peer-reviewed, open access journal focusing on the pathological basis of all cancers, potential targets for therapy and treatment protocols employed to improve the management of cancer patients. The journal also focuses on the impact of management programs and new therapeutic agents and protocols on patient perspectives such as quality of life, adherence and satisfaction. The manuscript management system is completely online and includes a very quick and fair peer-review system, which is all easy to use. Visit http://www.dovepress.com/ testimonials.php to read real quotes from published authors. 